\title{
Efficient Local Cloud-Based Solution for Diabetic Retinopathy Detection
}

\author{
Dayananda Pruthviraja, JSS Academy of Technical Education, Bengaluru, India \\ Anil B. C., JSS Academy of Technical Education, Bengaluru, India \\ Sowmyarani C. N., RV College of Engineering, Bengaluru, India
}

\begin{abstract}
Damage of blood vessels in retina due to diabetes is known as diabetic retinopathy. It is one of the one of the important origins of blindness for adults. Loss of vision can be avoided by detecting damage of retina (leaking fluid or blood). Efficient local cloud-based solution for diabetic retinopathy detection is designed in the work, where convolution neural network is used for training and classification module and achieved an accuracy of $86 \%$ using kappa metric. Fundus images are used for training and classification. System network architecture is derived from VGGNet. Network is trained using 80,000 images. Since everything is automated, a doctor is only required for treatment, not for diagnosis.
\end{abstract}

\section{KEYWORDS}

Convolution Neural Network, Diabetic Retinopathy, Fundus Image, Kappa Metric, VGGNet

\section{INTRODUCTION}

The diabetes problem associated with retina is Diabetic Retinopathy(NIH National Eye Institute, 2014). It can be avoided by detecting damage of retina (leaking fluid or blood). Loss of vision can be avoided by early detection of diabetic retinopathy.

It progress through four stages:

1. Mild nonproliferative retinopathy: A little amount of swelling near blood vessels of theretina which are called micro-aneurysms. This mainly occurs at a very early stage of the disease. Some amount of fluid leakage may also take place into the retina.

2. Moderate nonproliferative retinopathy: Increase in the swelling and distortion of theblood vessels due to their inability to transport blood. The retina's appearance can change and may lead to Diabetic Macular Edema.

3. Severe nonproliferative retinopathy: In this case, some blood vessels are completelyblocked thereby eliminating the blood supply to the areas near the retina. In some cases this may lead to growth of newer blood vessels near the retina.

4. Proliferative diabetic retinopathy (PDR): The most advanced stage of Diabetic Retinopathy is PDR where the proliferation of recently-grown blood vessels occurs. Some blood vessels also 
start to grow inside the surface of the retina, eventually turning into a gel-like patch, possibly filling the eyeball and causing blindness.

Convolutional Neural Network (CNN) is a branch of deep learning and has a massiveapplication in the field of image analysis and medical imaging for detection of disease. They are neural network architectures specifically designed for handling data with some spatial topology (e.g. images, sound, videos, character sequences in text, 3D voxel data etc). Convolutional Neural Network also has a collection of hidden layers. Specialized low power microscopic, indirect ophthalmoscopes capture fundus images (Kanski, J.J., Bowling, B., 2011), yielding high quality images in terms of resolution, clarity and contain a high pixel value. Fundus image is used in proposed work.

It is observed that there is a need of an automated detection system which can easily detect the presence or an absence of the disease and can also save time and effort that is wasted in the manual detection of diabetic retinopathy. Also the use of CNN (Andonová M., et al.,2017) in the detection of the disease by using the retinal images makes it an important approach towards the early detection of diabetic retinopathy(AndonováM.,et al.,.2017). The design of this system intentionally automates the entire diagnosis process to classify the level of diabetic retinopathy through use of the patient's fundus images.The ratio of people affected with the disease to the number of eye specialist who can screen these patients is very huge. Hence there is the need for an automated diagnostic system to measure diabetic retinopathic changes in the eye, so the affected personcan be referred to the specialist for further intervention and treatment,It has been observed that the whole process is manual and takes a lot of time to diagnose the disease so the initialObservations from ophthalmic practice reveals that this manual practice for diagnosis is time consuming. Also, using Convolutional Neural Network enables the large dataset of various classes of DR to serve as a foundation for further input of a real time dataset to diagnose specific patients' eye conditions and risk for blindness. As the dataset of the system receives more data, resulting in an oversampling of the image dataset, researchers can rebalance the population of the dataset, to increase the accuracy of diagnosis suggested by this local cloud-based solution for diabetic retinopathy detection. Eventually the accuracy of $96 \%$ is also a source of motivation of extending the modules to the next stage where other neural network models can also be included for better accuracy.

\section{Figure 1. Normal Retina VS Diabetic retinopathy}
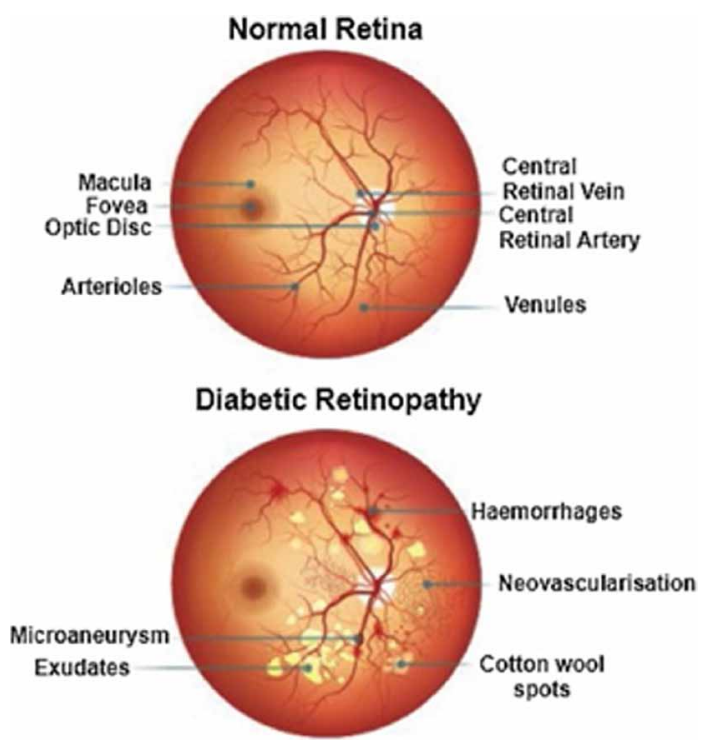
An automated diagnostic system for diabetic retinopathy detection, recognizing the change in an affected person's retina, through analysis of fundus images via the CNN architecture, facilitates further intervention and treatment via a timely referral to a specialist/retinologist. The system gives an automated way to detect this disease in its early phase using supervised learning methods to classify a given set of fundus images into 5 classes (0-4) based of the severity of the disease.

The main objectives of the system and its related results that are to be obtained after the successful training and classification of the dataset. Three specific objectives of this system are: (1) preprocessing the fundus image; (2) use Convolutional Neural Network to train/construct/embed the system; and (3) classify each image within cloud-based infrastructure.

\section{RELATED WORK}

This next section will share further details from prior research and practice to support the need for this automated diagnosis system for diabetic retinopathy detection.

The complexity of localizations and features of diabetic retinopathy create difficulty for patients and maybe for ophthalmologists. As a starting point for diagnosing this condition or disease, retinopathy fundoscopy is used to identify pathological findings.Due to presence of ruptured blood vessels, micro-aneursyms, capillaries small out pouching, hemorrhages on fundoscopic images easily can identify problems. To optimize the working nature of ophthalmologist'scomputerized diagnosis of diabetic retinopathy was used.Many computerized methods were used to detect micro-aneursyms to work with fundoscopic images of diabetic retinopathy. Patches of normal eye retina with blood cells Patches of normal eye retina without blood cells, retinas with micro-aneurysms compare to normal patches of eye retina initiallyis used with artificial neural networks to obtain difference between diabetic eye retina and normal human eye retina. Different types of features other than micro-aneurysms are required to identify the diabetic retinopathy, Many image processing techniques like low variance, various high bias methods are used to recognize a specific feature used in the identification of disease such as use of algorithm like top-hat algorithm for detection of micro-aneurysms.

Different method to detect micro-aneurysms and grading Diabetic Retinopathy involving k-nearest neighbor, ensemble based methods, Support vector machine and have yielded specificitiesand sensitivities and identified around 90\% range using various preprocessing techniques and feature extraction techniques. Earlier many studies on Convolution neural network for fundus images achieved the properties like specificities and sensitivities around in the range of 90 percent on huge datasets of around 80,000 to 120000 medical images to classification of normal and abnormal images. Abnormal images may be mildly or moderately or completely different nothing but severe changes from normal images. In this research paper the specificity and sensitivity of mild versusmoderateversus severe images are compared by using 4-ary classification model and performance are evaluated by comparing results with data augmentation technique and CNNs using binary classifiers by increasing the number of different classes to obtain an best results from diabetic retinopathy image (Lam Carson, Yi Darvin , Guo Margaret ,Lindsey Tony,2018).

Local binary Patterns are used for recognition of exudates and hemorrhages,some part of work has been planned to identify Diabetic Retinopathy by features of texture to protect Hemorrhages, hard Exudates, and micro-aneurysms, Around on 89 images database tests were performed and got an Area Under the Curve (AUC) of 0.87 and an normal accuracy of 86.15. To detect micro-aneurysms authors used fluroescein angiography(FA) images, even they used multi overlapping windows and radon transform to detect micro-aneurysms. Experimental tests were done on three types of databases like 120 images database,50 images database and 22 eye images database. Authors got results for specificity and sensitivity of $75 \%$ and $86 \%$ for the database of 120 images as best results compare to other two databases as mentioned above.micro-aneurysms can be effortlessly identified using modality of image, initially some injections are given to such kind of patients such that patient should not affect from any side effects by use of standard retinal image technologies of retinal can 
examine the retinal fundus imaging technology like discrete wavelet transforms are usedextraction of eight energy features were done six energy coefficients values in three different directions like vertical, diagonal and horizontal and other two features from the coefficients of two levels further support vector machines are used for categorization based on different kinds of kernels. Around 240 fundus images were in that around 120 are normal images and remaining with dissimilar degrees of Diabetic Retinopathy. For the polynomial kernel order of three using Support vector machine results are obtained for specificity, sensitivity and accuracy the results are more interesting but database size was very small.From recent yearsDiabetic Retinopathy detection is done by using deep learning techniques. These techniques required more images like big database for training here available public dataset is used for Diabetic Retinopathy detection (Chetoui Mohamed, et al., 2018).

Preprocessing and post processing steps are required for almost all existing algorithms to recognize the different stages of diabetic retinopathy, some algorithms requires manual interception to extract feature to identify the different stages in fundus images, in this research work no manual intervention is required to classify the different levels of diabetic retinopathy stages here an algorithm of Deep convolutional Neural Network is used which is completely automatic as intervention of doctor is not required at feature extraction stages. Significant classification accuracy is obtained from dropout techniques architecture, true positive rate also enhanced by using this dropout technique. Also there are many drawbacks among that first is for this architecture an one more stage is required that is augmentation stage is required for images captured from some special kinds of camera in dissimilar views, second is this dropout technique is more complex and requires high level graphics for computation intensive requiring to process the high resolution pictures when there are more images stacked.(Chandrakumar, Kathirvel,2016).

Experienced doctors are required to recognize the many features of fundus images including small features and also complex features this helps in the diagnosis of images of diabetic retinopathy but whereas this more cumbersome and takes more time to identify features either it may be small feature or complex features. In this research work a convolution neural network technique is used to recognize the diabetic retinopathy from set of images and classifies the accuracy based on severity of images having diabetic retinopathy here a type of network is used along withconvolution neural networkarchitecture and also to identify the complex features like haemorrhages, exudates and microaneurysms data augmentation is done. In this research work

High end graphics processor is used to recognize automatically without human intervention. This experiment is done on publicly available huge dataset and produced very accurate around $75 \%$ accuracy and $95 \%$ sensitivity best results as compare to other approaches to recognize the diabetic retinopathy(Pratt Harry, CoenenFrans, Broadbent Deborah M, Harding Simon P, LYalin Zheng,2016).

\section{IMPLEMENTATION AND RESULTS}

The correctness of the obtained result is analyzed here. The classifier gives the accuracy of $96 \%$ and the classification if between the range 0-4 i.e. five classes based on the training of the dataset. The system is a python implementation so it's first executed in the command prompt after which an input image is fed captured by fundus photography as input in the editor. If the image contains diabetic retinopathy of particular class, a graph depicting the probability of eye belonging to particular class is provided. Initially the image is fed through augmentation module, followed by training module for the model to be trained by different methods Convolutional neural network(GidarisSpyros,Komodakis Nikos,2016), image augmentation and supervised learning(Sermanet Pierre, et al.,2014). The images fed into the system was initially preprocessed and the image processing techniques like histogram equalization, resizing etc were applied and the fundus image was enhanced for further processing. After the preprocessing phase the images were undergone augmentation because the data was skewed due to overfitting(Srivastava Nitish, et al.,2014). The image dataset was collected after the data 
augmentation phase and then the training was done by setting the appropriate weights and feeding 80000 images in the Convolutional Neural Network with multiple layers.

Kappa Coefficient vary from -1 to 1 and it is obtained from a numerical test to identify the exactness of a classification, its fundamentally identifies how better the classification performed is classification do great job than the random. 1 indicates that the classification is significantly better and 0 indicates not better. Appropriate kappa number was used in the training of the dataset and the model was trained for multiple epochs. After that the classification was obtained and the images were classified according to the class of the image. The single model accuracy of the convolutional neural network is 0.423 on a quadratic weighted kappa metric (Ghosh R, GhoshK. and Maitra S,2017). Convolutional neural network gave the resulting classification with the accuracy of $86 \%$ and the final output was seen on the local server.

Python package in the system is a must to execute the modules of the system. The modules are executed with proper python syntax and the images are given as input. The images captured by the fundus image are highly cluttered and distorted. Hence, the detection of diabetic retinopathy is a challenging task. The training dataset is also deficient and requires heavy augmentation. In particular, the detection models need to be well-trained to detect class 3 and 4 (i.e Severe and NPR Diabetic retinopathy). The model takes roughly 5 minutes to classify images and is fast and prompt. Fig 2 shows precision v/s classification for proposed, VGGNet and GoogLeNet(Alban Stanford, Marco,2016). Datasetused can be found in Kaggle repository, https://www.kaggle.com/kirollosawad/ diabeticretinopathy16k300/downloads/diabeticretinopathy16k300.zip.

GoogLeNet is a 22 layers pretrained Convolutional neural network and number of parameters is less as compare to AlexNet CNN. Default Input size used in googlenet is 224 x 224 pixels. VGGnet is a deep convolutional network for object recognition developed and trained by Oxford's renowned Visual Geometry Group (VGG). On different ImageNet dataset this group achieved good performance as compared to other neural networks. This VGGnet makes an improvement over different neural networks like Alexnet. Multiple 3x3 kernel sized filters are used in VGGnet where as in Alexnet

Figure 2. Precision v/s classification for VGGNet and GoogLeNet

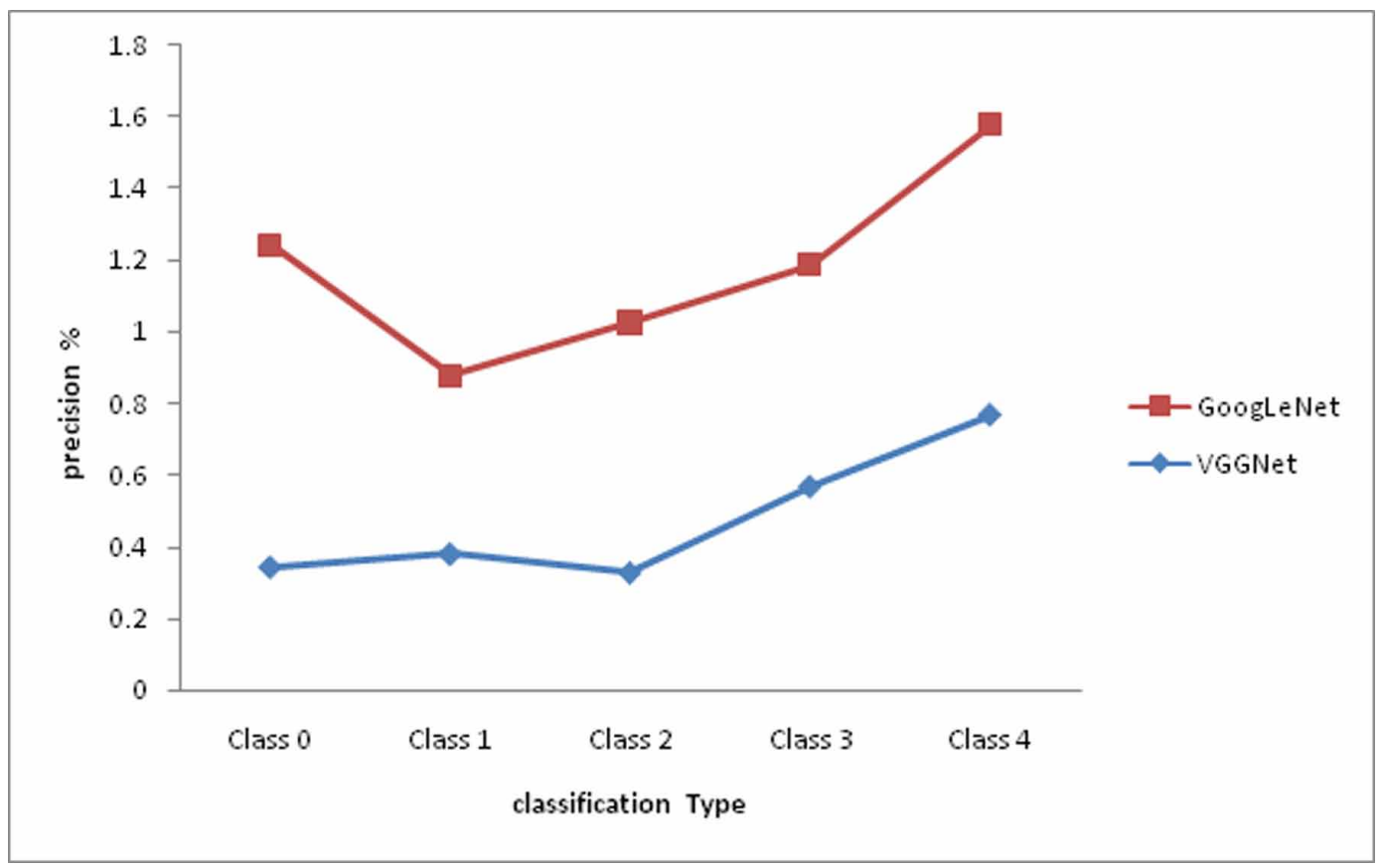


very large sized filters are used. In VGGnet multiple stacked kernel is used which gives the better performance than with the larger size kernel. The VGG neural net has divided into two layers: the "classifier" layer and the "future" layer. Future layer input is always an image of default size is 224 x 224 pixels.

An emerging field of research is automated medical imaging in this technology the disease is identified by the help of imaging technologies. In diabetic patients the diabetic retinopathy is identified mainly it is a retinal disease. To identify the diabetic retinopathy from fundus images deep neural network is used to classify the images. In this proposed technique many methods are used like principle component analysis (PCA), SoftMax, Gaussian mixture model (GMM), singular value decomposition (SVD) and GooglNet. Many experiments are done on KAGGLE datasets. This technique performance is better than spatial invariant feature transform (SIF) and Alexnet. Exponentially number of patients are increased from last few years as a result identification of diabetic retinopathy has also became a big challenge. For this challenge Deep neural network has been used to identify the DR in early stages. In this technique GoogleNet was used to classify the fundus images and other methods are used to decrease the dimensionality and to increase the reliability. More reliable and better architecture is GoogleNet which outperformed the results of Alexnet and SIFT methods. The accuracy of classification obtained from this proposed technique is 92.21\%.(Muhammad Mateen, Junhao Wen *, Nasrullah, Sun Song and Zhouping Huang ,2018).

\subsection{Constraint and Future Enhancements}

Apart from the accuracy of the model obtained, there are certain limitations that should not be ignored. These limitations are found in the data structure and the techniques used and can be taken as challenge for future enhancements are as follows:

- If the system is aided faster NVDIA Graphics Processing Unit version greater than P5000 and a larger training set, it could produce much better results in future

- The system is applicable for fundus image processing and cannot be through any simple photography techniques.

- Workstation is made as local server get services of application using Xammp

The future enhancements are, system could be enhanced to classify other generic eye diseases and system could be implemented with various different architectures such as ResNet, Inception V4 etc. last thing of extension can be, the system could also be implemented for mobile application where images can be captured from a high resolution camera and then it is fed into the application for detection of Diabetic Retinopathy .

\section{CONCLUSION}

The Convolution Neural Network used in the detection module and the classification module achieved a kappa metric accuracy of $96 \%$. The network architecture used in the system is all derived from the GoogleNet. The model will help cut down the time taken to diagnose from 10 days and multiple visits to the doctor to 1 visit and 15 minutes. The training of the networks has been done on 80,000 images. The developed system has reduced the detection time to approximately 5 minutes using fundus images. India has a large rural population without access to high-tech facilities or easy access to doctor. These are the most vulnerable class of people who generally disregard potential harms of diabetic retinopathy or are unable to gain medical attention. Through the implementation of this system time taken to diagnose is reduced to not more than 1 visit and since everything is automated a doctor is only required for treatment not diagnosis. The current work concentrates on setting up of local cloud and access it without any problem. 


\section{REFERENCES}

Andonová, M., Pavlovičová, J., Kajan, S., Oravec, M., \& Kurilová, V. (2017). Diabetic retinopathy screening based on CNN. International Symposium ELMAR, 51-54. doi:10.23919/ELMAR.2017.8124433

Carson, L., Darvin, Y., Margaret, G., \& Tony, L. (2018). Automated Detection of Diabetic Retinopathy using Deep Learning. AMIA Joint Summits on Translational Science Proceedings AMIA Summit on Translational Science, 2017, 147-155. PMID:29888061

Chandrakumar, T., \& Kathirvel, R. (2016). Classifying Diabetic Retinopathy using Deep Learning Architecture. International Journal of Engine Research, V5(06), 2016. doi:10.17577/IJERTV5IS060055

Coenen, Broadbent, Harding, \& Zheng. (2016). Convolutional Neural Networks for Diabetic Retinopathy. International Conference on Computing, Analytics and Security Trends (CAST). doi:10.1109/ CAST.2016.7914977

Divvala, Santosh, Ross, \& Ali.(2015). You Only Look Once: Unified, Real-Time Object Detection. IEEE Conference on Computer Vision and Pattern Recognition.

Ghosh, R., Ghosh, K., \& Maitra, S. (2017). Automatic detection and classification of diabetic retinopathy stages using CNN. 4th International Conference on Signal Processing and Integrated Networks (SPIN), 550-554. doi:10.1109/SPIN.2017.8050011

Jaderberg, Simonyan, Zisserman, \& Kavukcuoglu. (2016). Spatial Transformer Networks. In $13^{\text {th }}$ Conference on Neural Information Processing Systems. NIPS.

Kanski, J. J., \& Bowling, B. (2011). Clinical ophthalmology a systemic approach (7th ed.). Elsevier., doi:10.1016/ B978-0-7020-4093-1.00025-2

Karpathy. (2016). Connecting Images and Natural Language. Stanford University.

Mateen, M., Wen, J., Nasrullah, Song, S., \& Huang, Z. (2019). Fundus Image Classification Using VGG19Architecture with PCA and SVD. Symmetry, 11(1), 1. doi:10.3390/sym11010001

Mnih, Heess, Graves, \& Kavukcuoglu. (2016). Recurrent Modelsof Visual Attention. In $13^{\text {th }}$ Conference on Neural Information Processing Systems. NIPS.

Mohamed, Moulay, Akhloufi, \& Mustapha. (2018). Diabetic Retinopathy Detection Using Machine Learning and Texture Features. IEEE Canadian Conference on Electrical \& Computer Engineering (CCECE). doi:10.1109/ CCECE.2018.8447809

Nitish, , \& Geoffrey, , Krizhevsky, Sutskever, \& Salakhutdinov. (2014). Dropout: A Simple Way to Prevent Neural Networks from Overfitting. Journal of Machine Learning Research, 15, 1929-1958.

Sermanet, Eigen, Xiang, Mathieu, Fergus, \& Yann. (2014). Over Feat: Integrated Recognition, Localization and Detection using Convolutional Networks. Computer Vision and Pattern Recognition.

Spyros \& Nikos. (2016). LocNet: Improving Localization Accuracy forObject Detection. IEEE Conference on Computer Vision and Pattern Recognition (CVPR). doi:10.1109/CVPR.2016.92

Stanford, A. M. (2016). Automated Detection of Diabetic Retinopathy using Fluorescein Angiography Photographs. Stanford University.

Wei, Dragomir, Dumitru, \& Sz. (2016). SSD: Single Shot Multi Box Detector. Computer Vision and Pattern Recognition.

Xu, Feng, \& Mi. (2017). Deep Convolutional Neural Network-Based Early Automated Detection of Diabetic Retinopathy Using Fundus Image. Molecules, 22(12). .10.3390/molecules22122054 
Dayananda Pruthviraja is currently working as Professor \& HOD in the Department of ISE at JSSATE. He has Obtained Ph.D. degree from VTU and M. Tech degree from RVCE. His focus area is Image processing \& Information Retrieval. He was with MSRIT, Bengaluru, India in Department of ISE as an Assistant Professor. He has published international journals and conference papers in the field of Image processing \& Information retrieval.

Sowmyarani C. N. is working as an Associate Professor in Department of Computer Science and Engineering, RV College of Engineering, Bengaluru. She was with MSRIT Bengaluru in Dept. of CSE as an Assistant professor. She has published various international journal and conference papers in the field of Data Privacy and Information retrieval. 